\title{
Quench Characteristics of a Cu-Stabilized 2G HTS Conductor
}

\author{
E. A. Young, S. Chappell ${ }^{1}$, I. Falorio, Y. Yang
}

\begin{abstract}
The prospect of medium/high field superconducting magnets using 2G HTS tapes is approaching to reality with continued enhancement in the performance of these conductors. Direct measurements of $1 \mathrm{~d}$ adiabatic quench initiation and propagation of a $\mathrm{Cu}$-stabilized $2 \mathrm{G}$ conductor have been carried out with spatial-temporal recording of temperature and voltage following the deposition of various local heat pulses to the conductor at different temperatures between $40 \mathrm{~K}$ and $64 \mathrm{~K}$ carrying different transport currents. It was found that the stabilizer-free 2G tape maintains the unique characteristics previously measured in non-stabilized tape of increasing MPZ with transport current and higher quench energy at lower temperatures. The minimum quench energy, minimum propagation zone (MPZ) length are determined as a function of temperature and transport current. The change in MPZ size is investigated with measured temperature dependent E-J characteristics. The results add more detail to help understand the unique characteristics of increasing MPZ with transport current and lower temperatures.
\end{abstract}

Index Terms-Quench, thermal, electrical, superconducting.

\section{INTRODUCTION}

$\mathrm{O}$ ne of the principle concerns, once the critical current performance of a HTS superconductor tape is achieved is the thermal stability. HTS applications are targeted for temperatures from $4 \mathrm{~K}$ to $77 \mathrm{~K}$, but little is known about the affect on thermal stability of the temperature dependent current sharing and physical properties which occurs over this large temperature range. The ultimate goal of this on-going work is to improve the predictive capability of thermal stability so that performance requirements set out by applications can be related back to a desirable set of conductor properties.

The quench behavior of HTS at low temperatures is complex for the following reasons [1]-[4]. First of all, the current-share regime, fundamental to the development of the normal zone, is extended from just a few Kelvin above $T_{\mathrm{c}}$ for LTS to potentially $100 \mathrm{~K}$ for HTS at helium temperatures.

Manuscript received 3 August 2010. This work was supported in part by the U.K. Department for Trade and Industry under Grant IMPDAHAMA and cosupported by Oxford Instruments UK, Cobham Technologies..

E. A. Young, I. Falorio, Y. Yang are with the Institute of Cryogenics, School of Engineering Sciences, University of Southamtpon, UK. (+442380592661; fax +442380594767555-5555; e-mail:

e.a.young@soton.ac.uk).

${ }^{1}$ S.Chappell, is with Oxford Instruments Nanoscience, Tubney Woods, Abingdon, Oxfordshire, OX13 5QX, UK.
Within this extended current-share regime, the highly nonlinear E-J characteristics of HTS cannot be approximated by the classical critical state model, consequently the heat generation in the current share regime is a complex nonlinear function of temperature, instead of the linear dependence found in LTS. Secondly, the relevant thermal-electrical properties including resistivity, specific heat and thermal conductivity also exhibit a strong dependence on temperature in the extended current share regime. Such coupled nonlinearities have the potential to fundamentally change the behavior of the underlying heat diffusion process during the quench onset and subsequent propagation.

Regardless of the many underlying parameters, in the current experimental set-up there are only two parameters that can be changed, the temperature and the percentage of critical current. However although the percentage of critical current is independent of the temperature, the absolute value of current increases with lower temperatures and affects the dissipation levels.

Previous work, [5], looked at unstabilised 2G conductor, and found that the temperature dependence of minimum quench energy was opposite to that in LTS. The unsatbilised conductor was a good entrance-level study due to its low critical current, $37 \mathrm{~A}$ at $77 \mathrm{~K}$, but due to the low thermal conductivity the normal zone size was too small to be resolved.

This study looks at a $4 \mathrm{~mm}$ wide $\mathrm{Cu}$ stabilized conductor. In a first attempt to better separate the percentage of critical current from the temperature, a second sample is measured in field. This not only allows us to compare the same percentage of critical current which is the same absolute current value but at a different temperatures but also to extend the measurement range to lower temperatures that would otherwise be above the current injection limit of the apparatus.

\section{EXPERIMENTAL}

\section{A. Measurement}

Quench measurements were carried out in a cryocooled cryostat as described in [6]. The sample was thermally anchored at the two current contacts, which were connected via thermal links to the cryocooler head. The temperature of both contacts were controlled using a heater and can be varied between $20 \mathrm{~K}$ and $80 \mathrm{~K}$. A set of hybrid current leads were used for injecting transport current up to $250 \mathrm{~A}$ from room temperature to the current contacts. The high temperature ends of the HTS section were maintained at $77 \mathrm{~K}$ using a liquid 


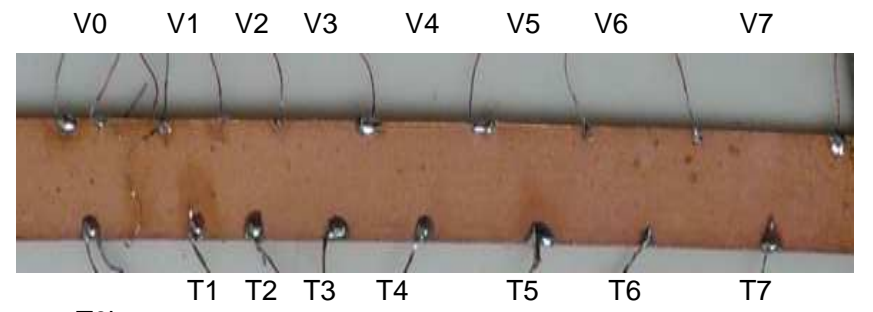

TOb
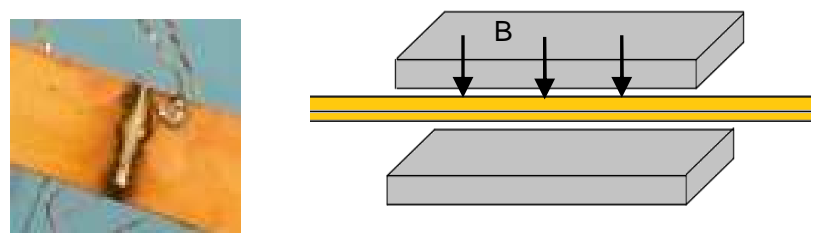

Fig.1. Sample mounted with spatially resolved temperature and voltage sensors (top). The heater, is mounted on the opposite side of the tape, (bottom left). Schematic of in-field sample mounted between two $50 \mathrm{~mm}$ long bar magnets (bottom right).

nitrogen buffer, which also provided cooling to a radiation shield. The cryogenic design was sufficient for maintaining a stable set temperature between $20 \mathrm{~K}$ and $80 \mathrm{~K}$ at the current injection levels throughout the measurements.

The process of quench initiation and propagation was measured using a set of spatially distributed voltage taps and miniature differential thermocouples soldered directly to the sample. The reference junctions of the differential thermocouples were thermally anchored at the current contacts. The electrical and thermal potentials were recorded simultaneously at up to $10 \mathrm{kHZ}$. The conductor was protected from burning out during quench by limiting the maximum output voltage of the current source and ultimately by a quench protection system to cut off the current source when the overall voltage across the sample exceeded a maximum level (typically $10-100 \mathrm{mV}$ ).

Localized thermal disturbances for quench initiation were applied using short heat pulses (10-100ms) discharged by a miniature heater made from conductive carbon loaded epoxy. The heater was attached to the conductor using silver-loaded epoxy, and on of the electrodes for the heater was on the sample. The electrical connectivity was used as a direct indication for the thermal connection between the sample and heater. The energy deposited was varied with a resolution up to $0.5 \%$ by changing the level and duration of the electrical pulse. Further details of the experimental procedure can be found in earlier publications [7-8].

\section{B. Samples}

The sample used is a standard $\mathrm{Cu}$ stabilized YBCO $2 \mathrm{G}$ conductor produced by Superpower Inc. As described in [9], Fig.1. shows a top view of thermal and electrical contacts. The heater is mounted on the underside of the tape, (Fig.1. bottom left).

A second sample was mounted between two Neodymium Iron Boron magnets in order to reduce the critical current. A clearance of $\sim 1 \mathrm{~mm}$ was maintained between the magnet face and the sensors on the sample surface. A 100 micron thick, $2 \mathrm{~mm}$ wide strip of G10 glass fiber was bonded with Epoxy to the tape surface to stiffen the tape so that a constant distance is maintained from the magnets surfaces.

\section{RESULTS \& DISCUSSION}

\section{A. Thermal-Electrical Properties}

The quench behavior of HTS at low temperatures is fundamentally influenced by its non-linear thermal-electrical properties over a wide temperature range where the current is shared between the "quenched superconductor" and the resistive matrix. As a precursor to the present study, the specific heat $c_{\mathrm{p}}$, thermal conductivity $k$, and resistivity $\rho$ were measured between $2 \mathrm{~K}$ and $300 \mathrm{~K}$, as shown in Fig.2. The normal resistivity (solid line) below $T_{\mathrm{c}}$ was obtained with a sample that was specially processed at $600^{\circ} \mathrm{C}$ in vacuum to disable the superconducting transition. The dashed line represents the superconducting transition at $88 \mathrm{~K}$ measured with the as received sample. Below $30 \mathrm{~K}$, the sample shows a residual resistivity of $0.22 \mathrm{n} \Omega \mathrm{m}$, i.e. RRR $\sim 160$. The resistivity is mainly attributed to the $20 \mu \mathrm{m} \mathrm{Cu}$ layer covering the YBCO film. The specific heat at low temperatures $(<6 \mathrm{~K})$ exhibits a significant linear electronic component attributed to $\mathrm{Ni}$ in the Hastelloy substrate. The thermal conduction is almost totally carried by the $\mathrm{Cu}$ layer with a large peak at $13 \mathrm{~K}$ confirming its high purity.

The in-field sample and self field sample critical is shown in Fig.3. Both decrease linearly with temperature. From the reduced critical current the field was estimated to be $\sim 0.1 \mathrm{~T}$, [9].

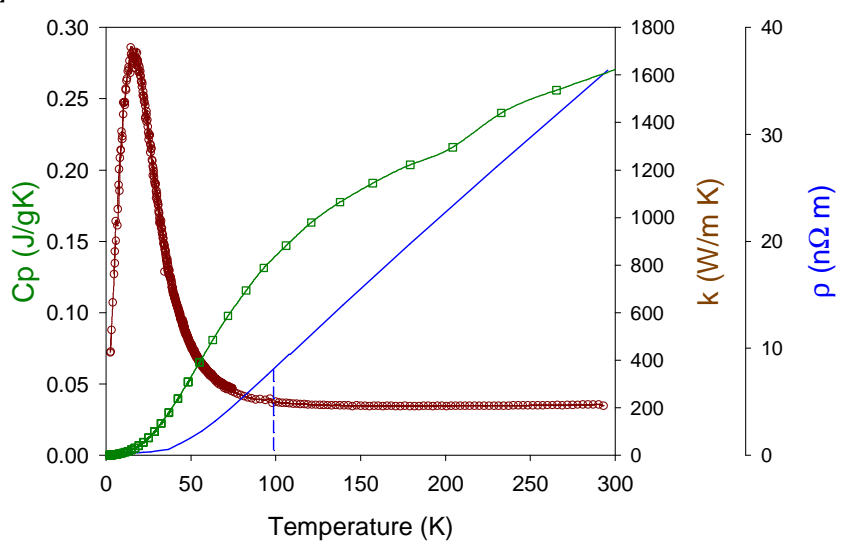

Fig. 2. The specific heat $(\square)$, thermal conductivity $(\bigcirc)$, and resistivity (solid line) of the $2 \mathrm{G}$ conductor at different temperatures.

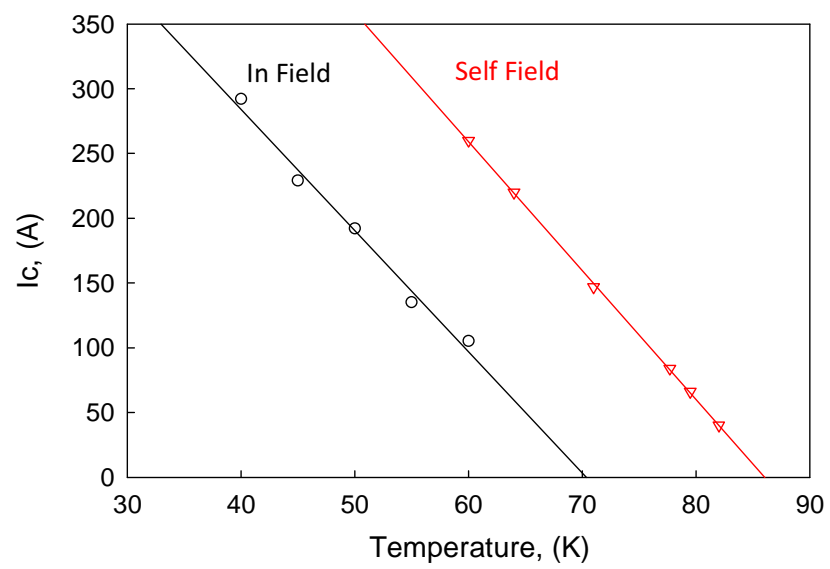

Fig.3. The temperature dependent critical current for the self-field and in-field samples. 


\section{B. Quench Initiation}

A measurement of the minimum quench energy at $64 \mathrm{~K}$, with $90 \%$ Ic is shown in Fig. 4. As shown previously this measurement is achieved after iterative incremental increases in the energy injected into the heater, until the minimum quench energy condition is found. The heat pulse was for $10 \mathrm{~ms}$ and the plot is synchronized to the beginning of the pulse. The time resolved rise and spreading of the normal zone ( $>$ Tshare $=66 \mathrm{~K}$ ) can be seen. V0 rapidly rises to a peak and

its subsequent decrease is followed by the rises in temperature that spreads as far as T5. The normal zone at this stage, before $\mathrm{V} 0$ begins to increase again encompasses $\mathrm{T} 0$ to $\mathrm{T} 4>66 \mathrm{~K}$, and V0 to V4. After $\sim 0.6 \mathrm{~s} \mathrm{~V} 0$ starts to rise again due to the contribution of heat generation by the transport current in the normal zone, and the dissipation spreads to V4 followed by rapid thermal quench propagating to V5 after more than 1 second.

The evolution of the normal zone can be described with spatial and temporal dimensions. The minimum propogation zone, $\mathrm{mpz}$, is defined as the dimension of conductor above the current sharing temperature. With respect to time, the mpz should be defined at the point where the heat generation and cooling are nearest to an equilibrium state, and this is indicated with a dashed line in Fig. 4. The time period for the quasi stable mpz has no definition to date. As the study of this parameter is very exploratory, we leave loosely define it as the time from the beginning of the pulse till the rapid quench propagation.

\section{MPZ Time Constant}

The minimum quench condition was measured for percentages of critical current from $80 \%$ to $99 \%$, for the sample in magnetic field at $40 \mathrm{~K}, 50 \mathrm{~K}$. The data for $\mathrm{V} 0$ and T0b is presented in Fig. 5. shows how the voltage and temperature at $\mathrm{T} 0 \mathrm{~b}$ and $\mathrm{V} 0$ depend on the $\%$ of Ic at $40 \mathrm{~K}$. As would be expected for lower percentages of critical current a higher temperature rise is required to achieve a minimum propagation condition. Taking the temperature at the $\mathrm{mpz}$ condition described in section $\mathrm{B}$, the temperatures are $17 \mathrm{~K}$ at $80 \%,(230 \mathrm{~A}$, Tshare $=5.8 \mathrm{~K}), 10.7 \mathrm{~K}$ at $90 \%,(258 \mathrm{~A}$, Tshare $=$ $2.8 \mathrm{~K}), 8.5 \mathrm{~K}$ at $95 \%,(273 \mathrm{~A}$, Tshare $=1.2 \mathrm{~K}), 7.3 \mathrm{~K}$ at $97 \%$, $(278 \mathrm{~A}$, Tshare $=0.7 \mathrm{~K})$, and $6.3 \mathrm{~K}$ at $99 \%$, (284A, Tshare $=0.1 \mathrm{~K}$ ). As would be expected the increasing temperature rise for lower percentages of critical current gives rise to higher voltages at V0, as a larger energy is required to overcome the temperature difference for quench. What is quite novel is the time evolution with \% Ic increasing as Ic is approached to periods as long as 8 seconds. Similar behaviour was observed at $50 \mathrm{~K}$ and can be described as the MPZ time constant. At $80 \%$ the value is $\sim 200 \mathrm{~ms}$ comparing with $\sim 50 \mathrm{~ms}$ for the unstablised $2 \mathrm{~mm}$ sample, [], at $40 \mathrm{~K}$. The time constant is very sensitive to the thermal environment and the heater energy, so although we believe the data to be a good representation, we fully realize that the time constant is limited by the resolution of temperature and heat.

The behaviour of the time constant is related to the thermal diffusivity, which in the case of the unstabilised is 10 times

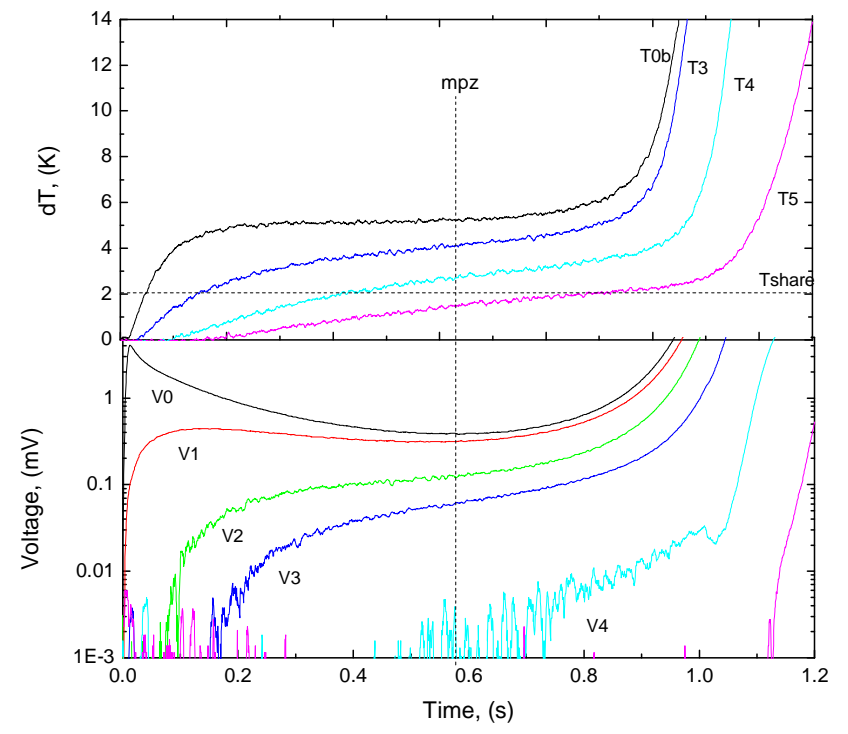

Fig. 4. The quench initiation at $64 \mathrm{~K}$ for the self-field sample. The temperature at which dissipation occurs, Tshare, and the time at which the mpz is taken are shown by dashed lines.

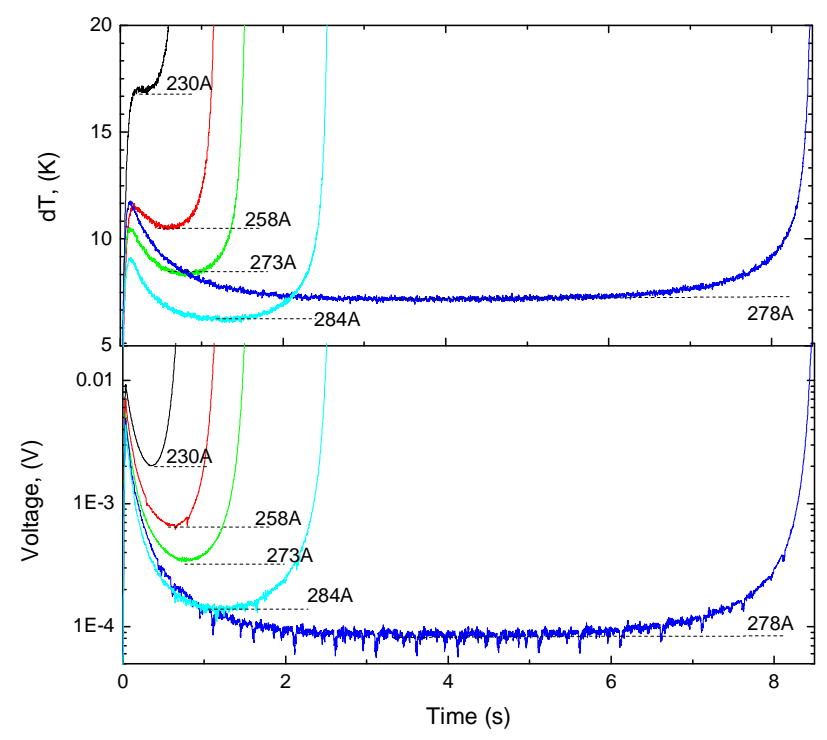

Fig 5. The change in quench onset with current at 40K shown with V0 and TOb.

smaller at $40 \mathrm{~K}$. Because of the rapid change in physical properties with temperature the behaviour of the time constant is dependent not only on the thermal diffusivity but the temperature rise required for a quench, which in turn depends on the critical current. For the stabilized sample due to the large temperature rise of $17 \mathrm{~K}$, which would be lower if Ic was higher, the thermal diffusivity reduces $\sim 4$ times at $57 \mathrm{~K}$.

\section{D. $M P Z$}

Taking the time at which the thermal propagation begins, as indicated in Fig. 4. for the self-field sample and the in-field sample, the mpz's are presented for $80 \%$ and 95\% Ic in Fig. 6. and Fig. 7. Horizontal dashed lines show the current sharing temperature, vertical dashed lines indicate the edge of the mpz. At $64 \mathrm{~K}$ the peak temperatures at $80 \%$ are $70.5 \mathrm{~K}$ and $95 \% 67.5 \mathrm{~K}$. The non-linear voltage dependence can be 


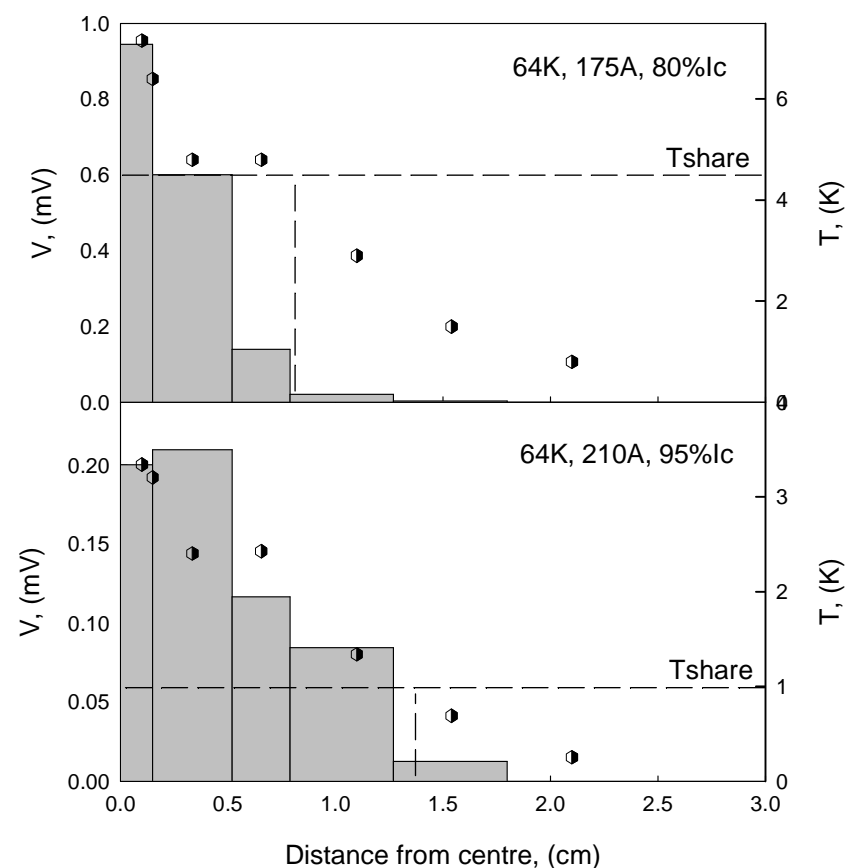

Fig, 6. The mpz described by temperature and voltage for the self-field sample.

observed from the correspondingly larger voltage of $0.95 \mathrm{mV}$ compared to $0.2 \mathrm{mV}$. The size of the $\mathrm{mpz}$ is however smaller for the larger voltage, $0.80 \mathrm{~cm}$ at $80 \%$ compared to $1.37 \mathrm{~cm}$ at $95 \%$. This may be surprising at first glance as one could expect the mpz size to increase with peak voltage. The increasing mpz size with \% Ic can be explained by the increased dissipation. With a larger heat flow from the center of the mpz the temperature gradient is steeper resulting in a narrower normal zone.

Looking at the in field $40 \mathrm{~K}$ data, the peak voltages are $2 \mathrm{mV}$ at $80 \%$, and $0.36 \mathrm{mV}$ at $95 \%$ with peak temperatures of $57 \mathrm{~K}$ and $48.5 \mathrm{~K}$ respectively. Comparing to the self field the dissipation is significantly larger at $40 \mathrm{~K}$, which would be expected as more heat is required to overcome the increased thermal conductivity which is twice as large. The size of the $\mathrm{mpz}$ is $1.25 \mathrm{~mm}$ at $80 \%$. At $95 \%$ the mpz has in fact grown beyond the measurement range available in the current set-up. The magnets only extend $2.5 \mathrm{~cm}$ from the centre of the sample, and this will have the effect of artificially reducing growth of the $\mathrm{mpz}$ as $2.5 \mathrm{~mm}$ is approached. What is clear is that the $\mathrm{mpz}$ at $95 \% \mathrm{Ic}$ is at least $2 \mathrm{~cm}$.

Comparing the in-field and self-field $\mathrm{mpz}$ sizes, the $\mathrm{mpz}$ can be seen to depend on temperature as well as percentage of critical current. The 1 dimensional analytical expression for the mpz length is

$$
l_{M P Z}=\pi \sqrt{\frac{k(\bar{T})\left(T_{Q}-T_{g}\right)}{G(\bar{T}, J)}},
$$

An increased mpz can be due to the larger thermal conductivity at $40 \mathrm{~K}$. There is however another possible explanation. The dissipation is determined by the $\mathrm{E}(\mathrm{J})$

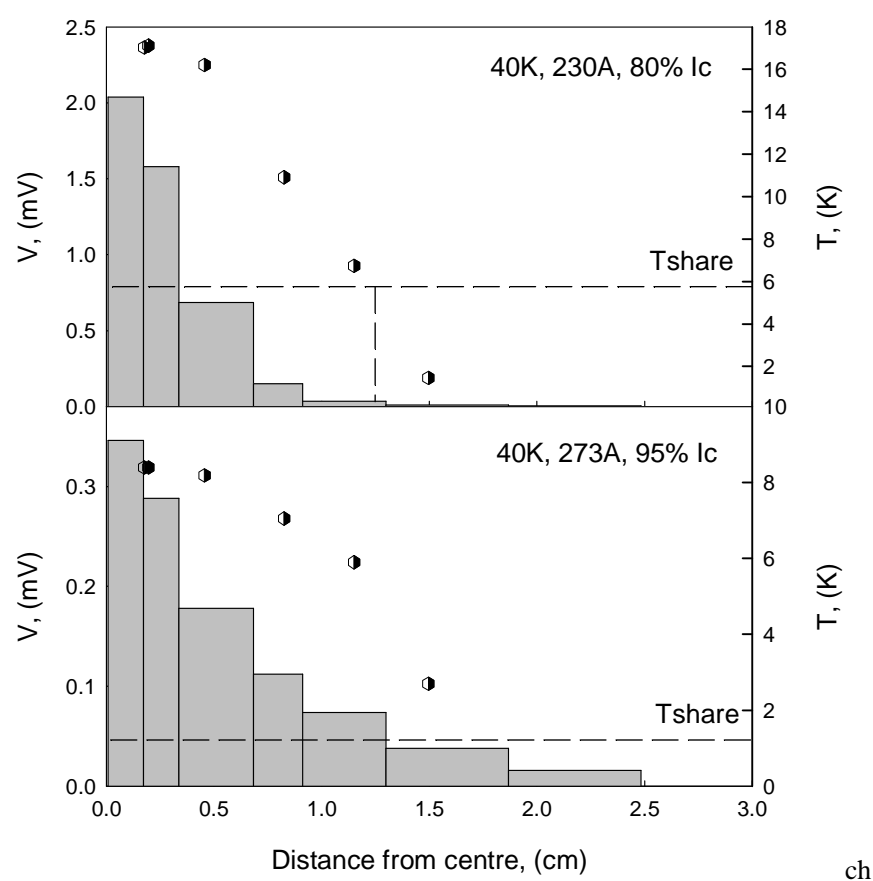

Fig, 7. The mpz described by temperature and voltage for the self-field sample.

characteristics which depends on the $n$ number. If the $n$ number is lower there will be less temperature dependence on the dissipation, $\mathrm{G}(\mathrm{T}, \mathrm{J})$, and from equation (1) this leads to a larger mpz.

\section{CONCLUSION}

Measurement of the quench initiation and propagation have been carried out on $\mathrm{Cu}$ stabilized $2 \mathrm{G}$ conductor from $40 \mathrm{~K}$ to $64 \mathrm{~K}$. The data captured shows the formation time and size of the normal zone before rapid quench. With increasing percentage of critical current both the size of the mpz and the time constant increase. Within a percentage points of Ic, this initial data shows the mpz and time constant behaviour to separate due to the time constant rapidly decreasing. The dependence of the mpz on temperature was seen more clearly by applying a magnetic field to decrease the critical current.

The data presented shows quite clearly the mpz increasing with lower temperatures. It is clear from the anlaysis that due to the rapid change in physical properties such as thermal conductivity and $\mathrm{E}(\mathrm{J})$ characteristics, detailed 1D modeling of the normal zone evolution is required to divulge which of the underlying parameters is responsible for the changes in $\mathrm{mpz}$ length. At this point the increased size of $\mathrm{mpz}$ with lower temperature and higher magnetic fields can be explained both by a smaller $\mathrm{n}$ number or larger thermal conductivity. Further work will concentrate on 1D modeling of this conductor and more systematic measurement in fields of 0 to $5 \mathrm{~T}$ across a wider range of temperatures.

\section{REFERENCES}

[1] M. N. Wilson, Superconducting Magnets, Oxford: Clarendon, 1983.

[2] I. Iwaza, Case Studies in Superconducting Magnets, New York: Kluwer Academic, 1994. 
[3] L. Dresner, Stability of Superconductors, New York: Kluwer Academic, 2002.

[4] R. B. Banks, Growth and Diffusion Phenomena: Mathematical Frameworks and Applications, Berlin: Springer, 1994.

[5] Edward A. Young, C. Friend, YiFeng Yang, "Quench characteristics of a stabilizer-free 2G HTS conductor", IEEE Trans on App. Supercon, vol.19, No. 3, June 2009, pp 2500-2503.

[6] T. Huang, A. Johnston, Y. Yang, C. Beduz, C. Friend, "Finite element modeling of thermal stability and quench propagation in a pancake coil of PbBi2223 tapes", IEEE Trans. App. Supercond., vol 15, June 2005 pp $1647-1650$.
[7] E. Martínez, F. Lera, M. Martinez-López, Y. Yang, S. I. Schlachter, P. Lezza, P. Kováč, "Quench development and propagation in metal/ $\mathrm{MgB}_{2}$ conductors" Supercond. Sci. Technol. vol. 19 Jan. 2006 pp 143-150.

[8] E. Martínez, E.A. Young, M. Bianchetti, O Munoz, S. I. Schlachter, Y.Yang, "Quench onset and propagation in $\mathrm{Cu}$-stabilized multifilament $\mathrm{MgB}_{2}$ conductors" , Supercon. Sci. Technol., Vol.21, 025009, (8pp) (2008).

[9] D. Turrioni, E. Barzi, M. J. Lamm, R. Yamada, A. V. Zlobin, A. Kikuchi, "Study of HTS Wires at High Magnetic Fields", IEEE Trans. App. Supercond., vol 19, No. 3, June 2009 pp $3057-3060$. 